\title{
Root Square Mean Labeling (RSML) of New Crown Graphs
}

\author{
R. Abdul Saleem, R. Mani
}

\begin{abstract}
A graph $G$ with a links and $b$ nodes is called RSMG if it is possible to label the links $x \in V$ with distinct labels $f(x)$ from $1_{0} 2_{w} q+1$ in such a way that when every node $e=u v$ is $f(e=u v)=\left\lceil\sqrt{\frac{f(u)^{2}+f(v)^{2}}{2}}\right\rceil$ or $\left[\sqrt{\frac{f(u)^{2}+f(v)^{2}}{2}}\right]$, then the resulting nodes labels are separate. In this case $f_{\text {is called a }}$ RSML of $G$. In this article we prove that some new crown graphs such as

$$
C_{n} \cup\left(P_{k} \odot K_{1}\right), C_{n} \cup\left(P_{k} \odot K_{2}\right)
$$$$
C_{n} \cup\left(P_{k} \odot K_{3}\right)_{0}\left(C_{n} \odot K_{1}\right) \cup\left(P_{k} \odot K_{1}\right)
$$

, $\left(C_{n} \odot K_{1}\right) \cup\left(P_{k} \odot K_{2}\right),\left(C_{n} \odot K_{1}\right) \cup\left(P_{k} \odot K_{3}\right)$ are root square mean graphs.
\end{abstract}

Keywords: Graph, RSML, Path, Cycle, crown.

\section{INTRODUCTION}

In this article, the graphs which are simple, countable and undirected with a links and b nodes. For a labeling graph we refer to Gallain [2]. For all other standard terminology and notations, we follow Harary [3].

The idea of RSML has been introduced by Sandhya, Somasundaram and Anusa in 2014 [8]. Some new results proved of Root Square Mean Labeling of Some Crown Graphs by R. Abdul Saleem and R. Mani [1]. In this article, we examine the RSML of New Crown graphs. Some new examples are presented and verified. We now give the definitions which are necessary for the present investigation.

\section{Definition 1.1:}

A graph $G$ with a links and b nodes is called RSMG if it is possible to label the links $x \in V$ with distinct labels $f(x)$ from 1,2 s $q+1$ in such a way that when every node $e=u v$ is $f(e=u v)=\left\lceil\sqrt{\frac{f(u)^{2}+f(v)^{2}}{2}}\right\rceil$ or $\left\lfloor\sqrt{\frac{f(u)^{2}+f(v)^{2}}{2}}\right]$, then the resulting nodes labels are separate. In this case $f$ is called a RSML of $G$. Definition 1.2:

A walk in which $u_{1} u_{2} \ldots u_{n n}$ are distinct is called a path. A path on $n$ vertices is denoted by $P_{n}$.

\section{Definition 1.3:}

A closed path is called a cycle. A cycle on $n$ vertices is denoted by $C_{\mathrm{m}}$.

\section{Definition 1.4:}

The two graphs $G_{1}=\left(V_{1}, E_{1}\right)$ and $G_{2}=\left(V_{2}, E_{2}\right)$ is a set $=E_{1} \cup E_{2}$.

\section{Definition 1.5:}

The Corona of graphs $G_{1}$ and $G_{2}$ is the graph $G=G_{1} \odot G_{2}$ formed by taking one reproduction of $G_{1}$ and

Revised Manuscript Received on December 5, 2019.

R. Abdul Saleem, Assistant Professor, Department of Mathematics, The Quaide Milleth College for Men, Chennai - 600100, Tamil Nadu, India.

R. Mani, Research Scholar, Department of Mathematics, Government Arts College, Chidambaram- 608102, India graph $G=G_{1} \cup G_{2}$ with link set $V=\mathbb{V}_{1} \cup V_{2}$ and the node

$\left\|V\left(G_{1}\right)\right\|$ reproductions of $G_{2}$ where the $i^{\text {th }}$ link of $G_{1}$ is adjacent to each link in the $i^{\text {th }}$ reproduction of $G_{2}$.

Definition 1.6:

Let $G_{1,} G_{2, m,} G_{n n}, n \geq 2$ be n replicas of a immovable graph $G_{x}$ The graph $G$ found by adding a node between $G_{\mathbb{I}}$ and $G_{1+1}$ for $i=1,2_{w} n-1$ is called a path union of $G$.

\section{MAIN RESULTS}

Theorem 2.1

Proof:

$$
C_{\mathrm{g}} \cup\left(P_{\mathrm{k}} \odot K_{1}\right) \text { is a RSMG. }
$$

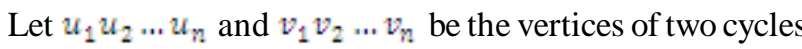
$C_{\mathrm{n}}$ in $G$.

Let $w_{1}=w_{1} w_{2} \ldots w_{k}=v_{1}$ be the vertices of the path $\mathbb{P}_{k}$.

Let $w_{1}^{\infty}, w_{2}^{0}, w_{\mathrm{n}}^{0}$ be the pendent vertices attached at $w_{1} w_{2} \ldots w_{k}$ respectively.

Let $G=C_{\mathrm{n}} \cup\left(P_{k} \odot K_{1}\right)$.Define a function $f_{m} V(G) \rightarrow\{1,2, \ldots, 3 n+k\}$ by

$f\left(u_{i}\right)=i+1$ for $1 \leq i \leq n-1$

$f\left(u_{n g}\right)=1$

$f\left(w_{i}\right)=n+k+i+4$ for $1 \leq i \leq n-1$

$f\left(v_{n i n}\right)=n+k+4$

$f\left(w_{i}\right)=n+2 i-2$ for $2 \leq i \leq k-1$

$f\left(w_{i}^{0}\right)=n+2 i+1$ for $1 \leq i \leq n-1$

Then clearly the nodes labels are separate.

Hence $f$ is a RSML of $G$.

Example 2.1.1:

The RSML of $C_{4} \cup\left(R_{*} \odot K_{1}\right)$ is given below:

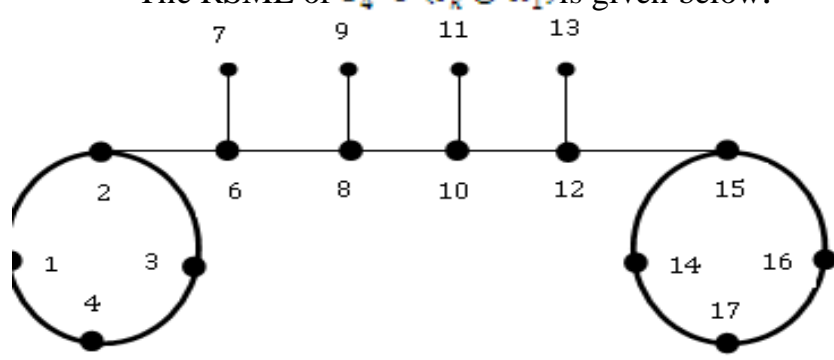

Figure 1

Theorem 2.2

$$
C_{\mathrm{g}} \cup\left(P_{\mathrm{k}} \odot R_{2}\right) \text { is a RSMG. }
$$

Proof:

Let $u_{1} u_{2} \ldots u_{n}$ and $\mathscr{V}_{1} \mathbb{V}_{2} \ldots \mathbb{V}_{n 2}$ be the links of two cycles $C_{\mathrm{n}}$ in $G$.

Let $w_{1}=w_{1} w_{2} \ldots w_{k}=v_{1}$ be the vertices of the path $P_{k}$.

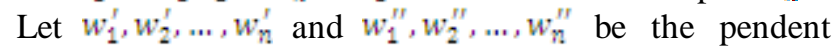
vertices attached at $w_{1} w_{2} \ldots w_{k}$ respectively.

Let $G=C_{\mathrm{m}} \cup\left(P_{k} \odot K_{2}\right)$. Define a function $f_{:}^{\prime \prime} V(G) \rightarrow\{1,2, \ldots, 4 n+k\}$ by

$$
\begin{array}{ll}
f\left(u_{i}\right)=i+1 & \text { for } 1 \leq i \leq n-1 \\
f\left(u_{n}\right)=1 &
\end{array}
$$

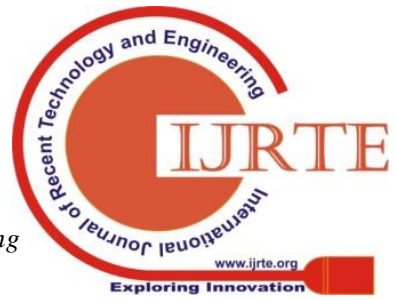


$f\left(v_{i}\right)=n+k+i+8$ for $1 \leq i \leq n-1$

$f\left(v_{n}\right)=n+k+8$

$f\left(w_{i}\right)=n+3 i-4 \quad$ for $2 \leq i \leq k-1$

$f\left(w_{i}^{i}\right)=n+3 i$ for $1 \leq i \leq n-1$

$f\left(w_{i}^{n}\right)=n+3 i+1$ for $1 \leq i \leq n-1$

Then clearly the node labels are separate.

Hence $f$ is a RSML of $G$.

Example 2.2.1:

The RSML of $C_{4} \cup\left(R_{x} \odot K_{2}\right)$ is given below:

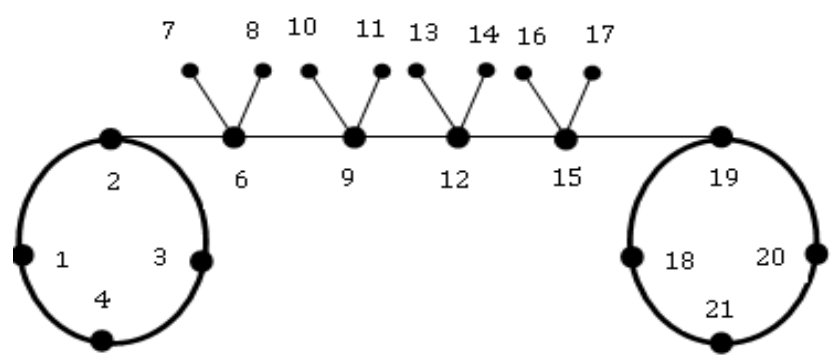

Figure 2

Theorem 2.3

Proof:

$C_{\mathrm{g}} \cup\left(P_{k} \odot K_{a}\right)$ is a RSMG.

Let $u_{1} w_{2} \ldots u_{n}$ and $\mathbb{v}_{1} \mathbb{V}_{2} \ldots \mathbb{V}_{n}$ be the vertices of two cycles $C_{\mathrm{n}}$ in $G$. Let $w_{1}=w_{1} w_{2} \ldots w_{k}=v_{1}$ be the vertices

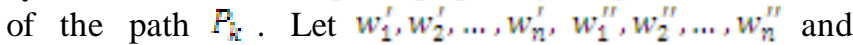

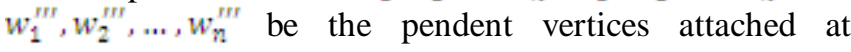
$w_{1} w_{2} \ldots w_{k}$ respectively.

Let $G=C_{\mathrm{g}} \cup\left(R_{\mathrm{k}} \odot R_{\mathrm{g}}\right)$. $\quad$ be a function $f_{:} V(G) \rightarrow\{1,2 \ldots \ldots, 5 n+k\}$ by

$$
\begin{aligned}
& f\left(w_{i}\right)=i+1 \text { for } 1 \leq i \leq n-1 \\
& f\left(u_{n}\right)=1 \\
& f\left(w_{i}\right)=n+k+i+12 \\
& 1 \leq i \leq n-1 \\
& f\left(w_{n}\right)=n+k+12 \\
& f\left(w_{i}\right)=n+4 i-6 \text { for } 2 \leq i \leq k-1 \\
& f\left(w_{i}\right)=n+4 i-1 \text { for } 1 \leq i \leq n-1 \\
& f\left(w_{i}\right)=n+4 i \text { for } 1 \leq i \leq n-1 \\
& f\left(w_{i}\right)=n+4 i+1 \text { for } 1 \leq i \leq n-1
\end{aligned}
$$

Then clearly the edge labels are distinct.

Hence $f$ is a RSML of $G$.

Example 2.3.1:

The RSML of $C_{4} \cup\left(R_{k} \odot K_{a}\right)$ is given below:

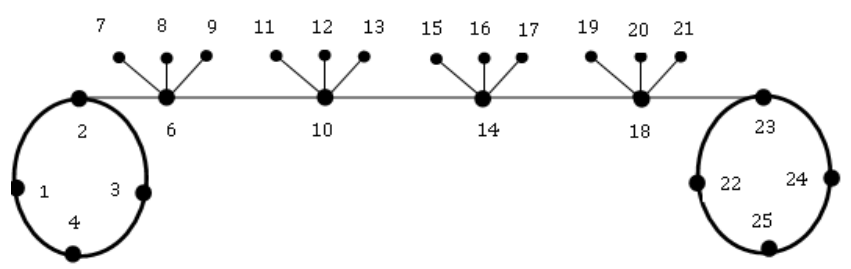

Figure 3

Theorem 2.4

$$
\left(C_{\mathrm{n}} \odot K_{1}\right) \cup\left(P_{k} \odot K_{1}\right) \text { is a RSMG. }
$$

Proof:

Let $\mathbb{U}_{1} \mathbb{u}_{2} \ldots \mathbb{u}_{n 2}$ and $\mathbb{V}_{1} \mathbb{V}_{2} \ldots \mathbb{V}_{n 2}$ be the vertices of two cycles $C_{\mathrm{n}}$ in $G$.

Let $w_{1}=w_{1} w_{2} \ldots w_{k}=v_{1}$ be the vertices of the path $P_{k}$.

Let $w_{1}^{\sharp}, w_{2, \ldots x}^{0} w_{\mathrm{n}}^{\sharp}$ be the pendent vertices attached at $w_{1} w_{2} \ldots w_{k}$ respectively.

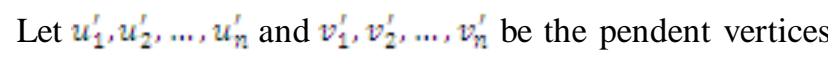

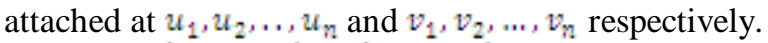

Let $G=\left(C_{\mathrm{s}} \odot K_{1}\right) \cup\left(P, \odot K_{1}\right)$.

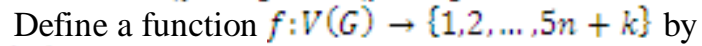

$f\left(u_{i}\right)=2 i+1$ for $1 \leq i \leq n-1$

$f\left(x_{n g}\right)=1$

$f\left(v_{i}\right)=2 n+k+2 i+4$ for $1 \leq i \leq n-1$

$f\left(v_{n g}\right)=2 n+k+4$

$f\left(w_{i}\right)=2 n+2 i-2$ for $2 \leq i \leq k-1$

$f\left(w_{i}^{i}\right)=2 n+2 i$ for $1 \leq i \leq n-1$

$f\left(u_{i}\right)=2 i+2 \quad$ for $1 \leq i \leq n-1$

$f\left(u_{i n}^{i}\right)=2$

$f\left(v_{i}^{i}\right)=2 n+k+2 i+5$ for $1 \leq i \leq n-1$

$f\left(v_{h}\right)=2 n+k+5$

Then clearly the edge labels are distinct.

Hence $f$ is a RSML of $G$.

\section{Example 2.4.1:}

The RSML of $\left(C_{4} \odot K_{1}\right) \cup\left(P_{k} \odot K_{1}\right)$ is given below:

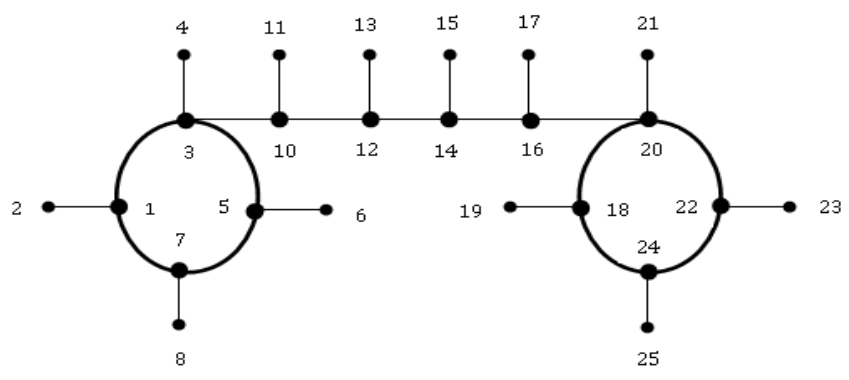

Figure 4

Theorem 2.5

$\left(C_{\mathrm{n}} \odot K_{1}\right) \cup\left(P_{k} \odot K_{2}\right)$ is a RSMG.

Proof:

Let $\mathbb{U}_{1} U_{2} \ldots u_{n}$ and $\mathbb{V}_{1} \mathbb{V}_{2} \ldots \mathbb{V}_{\mathrm{n}}$ be the vertices of two cycles $C_{\mathrm{n}}$ in $G$

Let $w_{1}=w_{1} w_{2} \ldots w_{k}=v_{1}$ be the vertices of the path $P_{k}$.

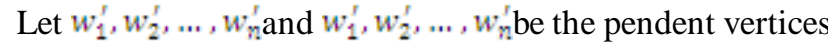
attached at $w_{1} w_{2} \ldots w_{k}$ respectively.

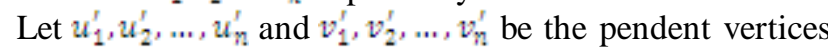

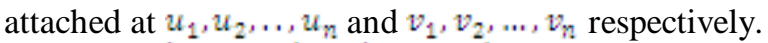

Let $G=\left(C_{\mathrm{s}} \odot K_{1}\right) \cup\left(P, \odot K_{2}\right)$.

Define a function $f_{m} V(G) \rightarrow\{1,2, \ldots n+k\}$ by

$$
\begin{array}{ll}
f\left(u_{i}\right)=2 i+1 & \text { for } 1 \leq i \leq n-1 \\
f\left(u_{n}\right)=1 & \\
f\left(v_{i}\right)=2 n+k+2 i+8 & \text { for } 1 \leq i \leq n-1 \\
f\left(v_{n}\right)=2 n+k+8 & \\
f\left(w_{i}\right)=2 n+3 i-4 & \text { for } 2 \leq i \leq k-1 \\
f\left(w_{i}\right)=2 n+3 i & \text { for } 1 \leq i \leq n-1 \\
f\left(w_{i}\right)=2 n+3 i+1 & \text { for } 1 \leq i \leq n-1 \\
f\left(u_{i}\right)=2 i+2 & \text { for } 1 \leq i \leq n-1 \\
f\left(u_{n}\right)=2 & \\
f\left(v_{i}\right)=2 n+k+2 i+9 & \text { for } 1 \leq i \leq n-1 \\
f\left(v_{n}\right)=2 n+k+9 &
\end{array}
$$

Then clearly the edge labels are distinct.

Hence $f$ is a Root Square mean labeling of $G$.

Example 2.5.1:

The RSML of $\left(C_{4} \odot R_{1}\right) \cup\left(P_{k} \odot K_{2}\right)$ is given below:

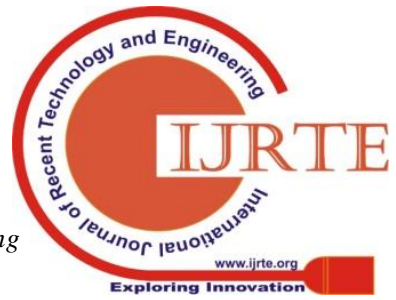




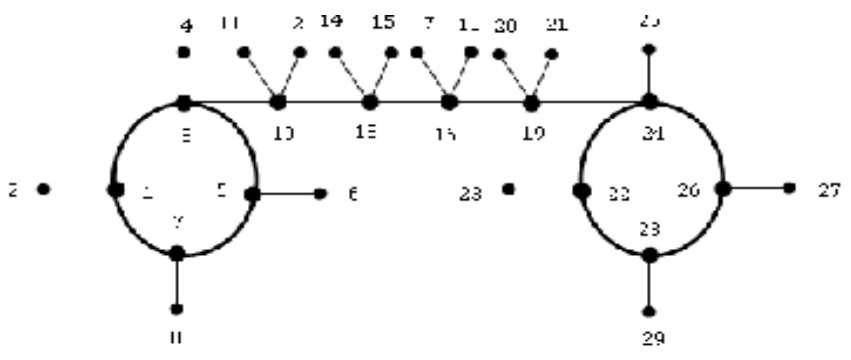

Figure 5

Theorem 2.6

$$
\left(C_{\mathrm{n}} \odot K_{1}\right) \cup\left(P_{k} \odot K_{\mathrm{a}}\right) \text { is a RSMG. }
$$

Proof:

Let $\mathbb{U}_{1} \mathbb{U}_{2} \ldots \mathbb{U}_{\mathfrak{n}}$ and $\mathbb{V}_{1} \mathbb{V}_{2} \ldots \mathbb{V}_{n \mathfrak{3}}$ be the vertices of two cycles $C_{\mathrm{n}}$ in $G$. path $\mathbb{P}_{\mathrm{k}}$.

Let $w_{1}=w_{1} w_{2} \ldots w_{k}=v_{1}$ be the vertices of the

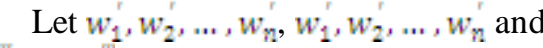
$w_{1}, w_{2}, \ldots, w_{n}$ be the pendent vertices attached at $w_{1} w_{2} \ldots w_{k}$ respectively.

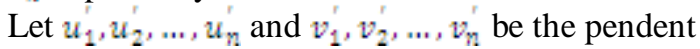
vertices attached at $\mathbb{U}_{1, x} \mathbb{U}_{2^{x, x},} \mathbb{u}_{n}$ and $\mathbb{V}_{1, x} \mathbb{V}_{2^{x}, x} \mathbb{V}_{h}$ respectively.

Let $G=\left(C_{\mathrm{n}} \odot K_{\mathrm{1}}\right) \cup\left(P_{\mathrm{k}} \odot K_{\mathrm{a}}\right)$.

Define a function $f_{u}^{\prime} V(G) \rightarrow\left\{1_{s} 2_{x}, 9 n+k\right\}$ by

$f\left(u_{i}\right)=2 i+1$ for $1 \leq i \leq n-1$

$f\left(u_{n n}\right)=1$

$f\left(w_{i}\right)=2 n+k+2 i+12$ for $1 \leq i \leq n-1$

$f\left(v_{n}\right)=2 n+k+12$

$f\left(w_{i}\right)=2 n+4 i-6$

for $2 \leq i \leq k-1$

$f\left(w_{i}\right)=2 n+4 i-1$ for $1 \leq i \leq n-1$

$f\left(w_{i}\right)=2 n+4 i$ for $1 \leq i \leq n-1$

$f\left(w_{i}\right)=2 n+4 i+1 \quad$ for $1 \leq i \leq n-1$

$f\left(u_{i j}\right)=2 i+2$ for $1 \leq i \leq n-1$

$f\left(u_{n}\right)=2$

$f\left(v_{i}\right)=2 n+k+2 i+13$ for $1 \leq i \leq n-1$

$f\left(v_{n}^{s}\right)=2 n+k+13$

Then clearly the edge labels are separate.

Hence $f$ is a RSML of $G$.

\section{Example 2.6.1:}

The RSML of $\left(C_{4} \odot K_{1}\right) \cup\left(P_{k} \odot K_{9}\right)$ is given below:

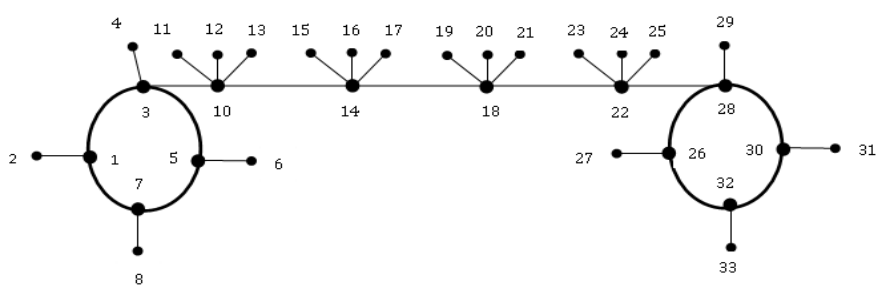

Figure 6

\section{CONCLUSION}

As all graphs are not RSMG's, it is very motivating to examine graphs which admits RSML. In this paper we prove that Path, Cycle, Crown are RSMG. Then, we present six new results on RSML of graphs. It is possible to investigate comparable results for numerous other graphs.

\section{REFERENCES}

1. Abdul Saleem. R and Mani. R, "Root Square Mean Labeling of Some Crown Graphs., The IJAEMA, V-XI, Issue X, Oct 2019, PP. 70-78.
2. Gallian. J.A, 2010, “A dynamic Survey of graph Labeling”. The EJC 17\#DS6.

3. Harary. F, 1988, "Graph Theory”, Nph Reading, New Delhi.

4. Meena. S and Mani. R, "RSML of Some Cycle Related Graphs., IJSART, Vol. 5, Issue 7, Jul 2019, PP. 786-789.

5. Sandhya. S.S, Somasundaram. S, Anusa. S, "Some Results on RSMG's" "JSR. Vol. 5, Issue 7, Jul 2019, PP. 786-789.

6. Sandhya. S.S, Somasundaram. S, Anusa. S, "Some More Results on RSMG,s" Communicated to "JSR". Vol.9, 2014.

7. Sandhya. S.S, Somasundaram. S, Anusa. S, "Further Results on RSML", "Bulletin of PAMS”. Vol.9, 2014, no.14, 667-676.

8. Sandhya. S.S, Somasundaram. S, Anusa. S, "RSMLG's" IJCMS, Vol.9, 2014. 